\title{
Cumplimiento de las Políticas Relacionadas con la Asepsia y Consumo de Antimicrobiano asociado a la Atención en Salud en un Hospital de Barranquilla
}

\section{CompliancewithPoliciesRelatedAsepsisAntimicrobial and Consumer of associatedHealthCare in a Hospital Barranquilla}

Recibido: 14 Mayo 2014/Enviado para modificación: 2 de Julio 2014/Aceptado: 23 de Octubre 2014

\author{
Edianis Acuña Zafra \\ Universidad del Atlántico \\ Boris Marchena Rubiano \\ Universidad del Atlántico \\ Guillermo Sarmiento Villa \\ Universidad del Atlántico \\ Oscar Camacho Romero \\ Universidad del Atlántico
}

\section{RESUMEN}

Introducción: Las infecciones asociadas con la atención en salud ocurren más de cuarenta y ocho horas después de la hospitalización y suelen considerarse como nosocomiales. Las que se presentan en el sitio donde se ha realizado una intervención quirúrgica también son frecuentes. Su incidencia varía de 0.5 a $15 \%$, según el tipo de operación y el estado subyacente del paciente. Objetivo: En este estudio se evaluó el consumo de antimicrobianos y las normativas de asepsia asociadas a las infecciones en un hospital del Sistema de Seguridad Social en Salud del AtlánticoColombia, tales como el cumplimiento del programa de control de infecciones, utilización de antimicrobianos, lavado de manos, limpieza y desinfección del entorno hospitalario en las áreas de cuidados intensivos y medicina interna. Resultados: Se encontró que el $42 \%$ de los pacientes prescriptos presentaban antimicrobianos como piperacilina + tazobactam, ampicilina sulbactam, ceftriazona, entre otros. También se halló resistencia de varios microorganismos responsables de infecciones nosocomiales, como escherichiacoli, pseudomonaaeruginosa y estafilococo coagulasa negativa; y, por último, se registró que el cumplimiento del uso de equipo de protección personal fue superior al 90\%. Conclusiones: Existe un cumplimiento de las condiciones de asepsia en la institución; no obstante, los registros indican la presencia de microorganismos, entre los más frecuentes están escherichiacoli y pseudomonaaeuroginosa. En cuanto a protección personal, la UCI plena presentó un $71.6 \%$ de cumplimiento mayor a las otras áreas evaluadas.

Palabras Clave:Antimicrobiano, asepsia, infección hospitalaria (Fuente: DeCS).

\section{ABSTRACT}

Introduction: Infections associated with health care occurs more than forty-eight hours after often considered Nosocomial hospitalized. Site infections surgery are also frequent the incidence varies from 0.5 to $15 \%$, depending on the type of operation and the patient's underlying condition. Objective: In this study we evaluated the consume of antimicrobial policies and regulations associated aseptic infections Hospital System in Health and Social Security of the Atlantic - Colombia, such as compliance with infection control program, use of antimicrobials, washing hands, cleaning and disinfection of the hospital environment in the areas of Intensive Care and Internal Medicine. Results: There is knowledge of nosocomial infections , also found that $42 \%$ of patients showed antimicrobial prescribed piperacillin + tazobactam, ampicillin- sulbactam, ceftriaxone, among others. They encountered resistance from several microorganisms responsible for nosocomial cases as Escherichia coli, pseudomonaaeruginosa and Staphylococcus coagulase negative, it was recorded that compliance with the use of personal protective equipment was over $90 \%$. Conclusions: There is a fulfillment of aseptic conditions in the institution and records exist which found that the most frequent microorganisms were Escherichia coli and pseudomonaaeruginosa, in full personal protective ICU presented a $71.6 \%$ compliance greater than the other areas assessed.

Keywords:Antimicrobial, asepsis, crossinfection(Source: MeSH, NLM).

Para citar este artículo: Acuña Zafra E, Marchena Rubiano B, Sarmiento Villa G, Camacho Romero O. Cumplimiento de las Políticas Relacionadas con la Asepsia y Consumo de Antimicrobiano asociado a la Atención en Salud en un Hospital de Barranquilla.Cienc. innov. salud. 2014; 2 (2):70 - 75 


\section{Introducción}

Las Infecciones Intrahospitalarias (IIH) representan un gran problema de salud pública mundial, tanto en las instituciones de salud de países desarrollados como en los que están en vía de desarrollo. Se trata de una realidad frecuente, tangible y mensurable, que evidencia la necesidad de realizar estudios descriptivos con el fin de establecer bases para futuras estrategias de intervención eficaz, es decir, que permitan la disminución de las tasas de morbimortalidad y, por supuesto, la disminución de costos innecesarios $(1,2)$.

Por su parte, las Infecciones Asociadas a la Atención en Salud (IAS) son aquellas sobre las cuales no existe registro de su presencia o estado de incubación cuando el paciente ingresa al servicio de salud y que se hacen evidentes o se manifiestan después de 48 horas de producida la admisión. Se toma por referencia 48 horas, pues tal es el periodo de incubación típico de los microorganismos (3).

Ahora bien, el vocablo nosocomial proviene del griego nosokomein que significa nosocomio, u hospital, y que, a su vez, deriva de las palabras griegas nosos, enfermedad, y komein, cuidar, o sea, "donde se cuidan enfermos" $(4,5)$.

En Estados Unidos, las IAS están entre las 10 primeras causas de muerte (6). El Instituto Americano de Medicina estima que efectos adversos prevenibles en los pacientes, incluyendo las IAS, son responsables de 44000 a 98000 muertes anuales en ese país, y que ello significa un costo de $\$ 17-\$ 29$ billones de dólares (7). En México los costos asociados con IAS en Unidades de Cuidados Intensivos (UCI) pediátricas implican una estancia adicional de los niños infectados de 9.6 días hospitalización, hecho que afecta el costo promedio por infección, calculado aproximadamente en \$ 12000 dólares (8).

Los pacientes de UCI se encuentran entre los más propensos a adquirir este tipo de infecciones, ya sea por su condición fisiológica o porque la mayoría de veces son sometidos a procedimientos invasivos, tales como catéter venoso central, sonda vesical, respirador mecánico, etc. (9), los cuales, al traspasar las barreras de defensa del organismo, facilitan la aparición de las IN (10).

Las UCIs son así unos de los servicios que mayor incidencia tienen en la aparición de las IAS. Por tal motivo, esta investigación buscó determinar el consumo de antimicrobianos y el cumplimiento de las normativas de asepsia relacionados con IAS en un hospital del área metropolitana de Barranquilla.

\section{Materiales y Métodos}

Se realizó un estudio descriptivo que permitió indagar sobre el cumplimiento de las políticas antimicrobianas y de las normativas de asepsia asociadas con las infecciones nosocomiales en las unidades de medicina interna y cuidados intensivos de un hospital de tercer nivel del área metropolitana de Barranquilla. Los sujetos de estudios fueron profesionales de la salud (médicos y enfermeras), que tenían contacto directo con el paciente y que laboran en las jornadas de mañana y tarde en las áreas evaluadas.

Se realizó una recolección prospectiva durante los meses de diciembre 2008 y abril 2009. Con el fin de obtener una mayor legibilidad, consistencia y totalidad de la información recolectada, se elaboraron formatos de evaluación, lográndose la evaluación individual de los factores e indicadores que intervienen en el cumplimiento de las políticas de antimicrobianos y normativas de asepsia. En la planificación de la gestión investigativa, se realizaron las diligencias pertinentes para la consecución del aval requerido para la realización de la investigación en la institución hospitalaria (11).

La recolección de la información implicó la utilización de tres estrategias. En primera medida, se realizaron entrevistas abiertas al personal clínico de las áreas de microbiología y UCI, con las que se indagó la forma cómo se realiza la vigilancia epidemiológica de las infecciones, esterilización y desinfección de alto nivel, ambiente hospitalario y saneamiento. La segunda estrategia se basó en la recolección de datos, a través de una guía de observación en los servicios hospitalarios. Esta permitió obtener la información sobre los procesos de asepsia de la institución.

Así mismo, se revisaron las historias clínicas de los pacientes hospitalizados en los servicios estudiados para considerar el sexo del paciente, unidad de ingreso, diagnóstico principal al ingreso, estancia y/o número de días que permaneció en la unidad, antibióticos administrados (frecuencia de prescripción), microorganismo aislado, sensibilidad y resistencia de microorganismos según antibiogramas, cumplimiento de los parámetros a seguir en el correcto cumplimiento de desechos generales evacuados y desechos infecciosos, seguimiento del cumplimiento diario de las normativas de asepsia y ambiente del

Cienc. innov. salud. Diciembre 2014; 2 (2):70 - 75. Universidad Simón Bolívar (Col).ISSN: 2344-8636 
asociado a la Atención en Salud en un Hospital de Barranquilla

hospital aun cuando se hallaba visiblemente limpio. Los datos se presentan en tablas de contingencia y distribución de frecuencias para una mayor comprensión.

\section{Resultados}

Durante el proceso de recolección de los datos suministrados, se involucraron médicos, enfermeras de las áreas de microbiología, esterilización y limpieza del entorno hospitalario, así como personal encargado del manejo de los desechos y otros recursos del centro hospitalario.

La población atendida comprendió $51.9 \%$ pacientes de sexo femenino y $48.1 \%$ pacientes de sexo masculino. Estos correspondieron a 81 pacientes en total, distribuidos en las áreas de medicina interna y cuidados intensivos. No se halló una diferencia marcada entre los pacientes de cada área evaluada.

Con relación a las políticas para el uso de antimicrobianos, la revisión de las historias clínicas mostró que al $42 \%$ de la población se le prescribió antimicrobianos para tratar diferentes tipos de patologías en otras áreas de estudio (Tabla 1). En este sentido, se evaluaron los pacientes que fueron ingresados a las áreas de medicina interna y UCI, observándose que presentaron prescripciones con algún antimicrobiano como piperacilina + tazobactam, ampicilina y sulbactam, los cuales son conocidos como inhibidores de las betalactamasas (12). Ahora bien, del total de pacientes prescritos con antimicrobianos en las áreas de medicina interna y cuidados intensivos en el periodo de estudio, según las historias clínicas, se encontró una tasa de infección nosocomial del $17.6 \%$ diagnosticadas por el médico tratante. Las áreas donde se presentaron mayores casos de infecciones intrahospitalarias fueron: en UCI plena, $8.8 \%$ de infecciones nosocomiales, medicina interna femenino, $5.9 \%$ y medicina interna masculino, $2.9 \%$. UCI Intermedios no arrojó resultados durante el periodo estudiado. Se evidencia, entonces, una presencia de infecciones en ambas áreas de medicina interna, pero con un mayor porcentaje en el área de UCI plena, lo cual puede estar relacionado con el tiempo de permanencia mayor de los pacientes en esta área en comparación con las otras evaluadas (Tabla 1).
Tabla 1. Características de los pacientes hospitalizados y uso de antimicrobianos en las áreas estudiadas según el sexo

\begin{tabular}{ccccccc}
\hline & \multicolumn{2}{c}{ Medicina Interna } & \multicolumn{2}{c}{ UCl Intermedia } & \multicolumn{2}{c}{ UCI Plena } \\
\cline { 2 - 7 } & Femeninı & Masculinc & Femenino & Masculino & Femenino & Masculin \\
\hline $\begin{array}{c}\text { Pac. Hospital- } \\
\text { lizados }\end{array}$ & $25,9 \%$ & $28,4 \%$ & $18,5 \%$ & $7,4 \%$ & $7,4 \%$ & $12,3 \%$ \\
$\begin{array}{c}\text { Prescripción } \\
\text { de Anti- } \\
\text { microbianos }\end{array}$ & $6,2 \%$ & $13,6 \%$ & $8,6 \%$ & $2,5 \%$ & $4,9 \%$ & 6,2 \\
$\begin{array}{l}\text { Infección intra- } \\
\text { hospitalaria }\end{array}$ & $5,9 \%$ & $2,9 \%$ & $0 \%$ & $0 \%$ & $2,9 \%$ & $5,9 \%$ \\
\hline
\end{tabular}

Fuente: Archivos de la Institución de Salud.

Los microorganismos aislados que se presentaron en las diferentes áreas fueron escherichiacoli (37.5\%), pseudomonaaeruginosa (25\%), estafilococos coagulasa (25\%) y proteusmirabilis $(12.5 \%)$. Para el caso de los microorganismos responsables de las infecciones intrahospitalarias, según los antimicrobianos, es pertinente lo relacionado en la Tabla 2.

Al examinar, los microorganismos responsables de IN según los antibiogramas, se halla que la sensibilidad más representativa se encuentra en la amikacina y gentamicina (10\%), cefepime, ciprofloxacina, meropenem, piperacilina + tazobactam y vancomicina $(7.5 \%)$.

Tabla 2. Sensibilidad de microorganismos responsables de Infecciones nosocomiales en las áreas de estudio

\begin{tabular}{|c|c|c|c|c|}
\hline \multirow[b]{2}{*}{ Antibiótico } & \multicolumn{4}{|c|}{ Microorganismo } \\
\hline & $\begin{array}{c}E . \\
\operatorname{coli}(\%)\end{array}$ & $\begin{array}{c}P . \\
\text { aeruginosa } \\
(\%)\end{array}$ & $\begin{array}{c}E . \\
\text { coagulasa } \\
\text { negativa } \\
(\%)\end{array}$ & $\begin{array}{c}\text { Total } \\
(\%)\end{array}$ \\
\hline Amikacina & 7.5 & 2.5 & - & 10 \\
\hline Gentamicina & 7.5 & 2.5 & - & 10 \\
\hline Cefepime & 2.5 & 5 & - & 7.5 \\
\hline Ciprofloxacina & 5.0 & 2.5 & - & 7.5 \\
\hline Meropenem & 2.5 & 5 & - & 7.5 \\
\hline $\begin{array}{l}\text { Piperacilina + } \\
\text { tazobactam }\end{array}$ & 2.5 & 5 & - & 7.5 \\
\hline Vancomicina & - & 2.5 & 5 & 7.5 \\
\hline Aztreonam & 5 & - & - & 5 \\
\hline Claritromicina & - & 2.5 & 2.5 & 5 \\
\hline Clindamicina & - & - & 5 & 5 \\
\hline Nitrofurantoina & 5 & - & - & 5 \\
\hline Cefalotina & 2.5 & - & - & 2.5 \\
\hline Ceftacidime & 2.5 & - & - & 2.5 \\
\hline Cefotaxime & 2.5 & - & - & 2.5 \\
\hline Ceftriaxona & - & 2.5 & - & 2.5 \\
\hline $\begin{array}{l}\text { Ampicilina + } \\
\text { sulbactam }\end{array}$ & - & - & 2.5 & 2.5 \\
\hline Norfloxacina & 2.5 & - & - & 2.5 \\
\hline Oxacilina & - & - & 2.5 & 2.5 \\
\hline Linezolid & - & - & 2.5 & 2.5 \\
\hline Rifampicilina & - & - & 2.5 & 2.5 \\
\hline $\begin{array}{l}\text { Total } \\
\text { antibióticossensibles }\end{array}$ & 47.5 & 30 & 22.5 & 100 \\
\hline
\end{tabular}

Fuente: Archivos de la Institución de Salud. 
Los recursos para el lavado de manos comprenden: abundante agua potable, uso de jabón o jabón antiséptico, uso de solución alcohólica, uso de cepillo para uñas, toalla de papel desechable, cerrado de llave con toalla desechable, tiempo adecuado, remoción de anillos, relojes y pulseras; en general, se encontró un cumplimiento significativo al respecto en todas las áreas estudiadas.

Para el seguimiento diario de los indicadores referidos al equipo de protección personal y/o precaución de aislamiento, se evaluó el uso de gorros, gafas aislantes, mascarilla, bata, zapatos y guantes, que se efectuó en las áreas y jornadas (mañana y tarde). Concretamente, en medicina interna, caballeros y damas, se cumplió en 45.7 y $45.9 \%$ respectivamente; para UCI Intermedia, fue de $65.5 \%$; mientras que UCI Plena fue el área con mayor porcentaje alcanzado, con $71.6 \%$.

Entre los antimicrobianos más utilizados, se encontraron: Piperacilina + tazobactam (17.4\%), ampicilina sulbactam y ceftriazona (14.5\%) y vancomicina (8.7\%). Por su parte, los microorganismos aislados, evaluados en relación al sexo, presentaron una tasa común del $8.8 \%$ de IN. Los agentes causantes de infección nosocomial fueron: Escherichiacoli (50\%), pseudomonaaeuroginosa (33.3\%), estafilococo coagulasa negativa (16.7\%), mostrando todo ello una clara representación de dichas infecciones por escherichiacoli en el área de M.I. Damas (33.3\%) y UCI plena (16.7\%). Con base en los antibiogramas hechos por el laboratorio clínico, en la resistencia de los microorganismos predominó la resistencia de la escherichiacoli y pseudomonas a trimetropinsulfa $(22.7 \%)$, así como la ampicilina + sulbactam (13.6\%).

En cuanto a la existencia de un comité para la vigilancia de las infecciones nosocomiales, el $40 \%$ del personal de salud del hospital hizo énfasis en que está conformado, pero es necesario fortalecerlo con el sector académico. Además, todo el personal entrevistado relacionó la definición de infecciones nosocomiales o la correlación directa con infecciones intrahospitalarias.

Mediante observación directa y entrevistas para la recopilación de información de tipo organizacional, estructural y técnica, se observó que en el campo microbiológico, se siguen todas las instrucciones y recomendaciones establecidas por las normas y leyes requeridas y que la identificación de los agentes microbianos más relevantes en el control de infecciones nosocomiales es llevada y seguida en las historias clínicas de los pacientes.

Durante la inspección, también se apreció que se utilizaban equipos como autoclave para el proceso de esterilización. Las normas y procedimientos se encuentran estandarizadas, entre estas se destaca la Bowie Dick, en la que se distribuye el vapor dentro del equipo; para garantizar sus resultados, se utilizan tirillas por paquetes de ropas.

Además, se encontró el hospital limpio, sin muestras de polvo ni suciedad, con registros de procesos de limpieza 3 veces por día y, cuando es necesario, en casos de derrames de fluidos corporales u otro líquido, así como en el momento que los pacientes abandonan la institución; mientras que en medicina interna, se halló que la desinfección es realizada semanalmente. Las ubicaciones de las canecas con sus respectivas bolsas, baños aseados se encontró en orden, así como lavamanos adecuados con abundante agua. En las áreas de UCI (plena e Intermedia), se encontró jabón líquido, alcohol antiséptico y toallas desechables para el secado de manos.

En la Tabla 3, se aprecia el porcentaje de resistencia de los microorganismos aislados responsables de infecciones nosocomiales en el centro hospitalario según los antibiogramas realizados, predominando trimetropinsulfa $(22.7 \%)$, ampicilina + sulbactam $(13.6 \%)$, ampicilina, cefuroxima y eritromicina (9.1\%).

Tabla 3. Resistencia de microorganismos responsables de infecciones nosocomiales

\begin{tabular}{|c|c|c|c|c|}
\hline \multirow[b]{2}{*}{ Antibiótico } & \multicolumn{4}{|c|}{ Microorganismo } \\
\hline & $\begin{array}{c}\text { E.coli } \\
(\%)\end{array}$ & $\begin{array}{c}\text { P.aerugino } \\
\text { sa }(\%)\end{array}$ & $\begin{array}{c}\text { Estafilococo } \\
\text { coagulasa } \\
\text { negativa }(\%)\end{array}$ & $\begin{array}{c}\text { Total } \\
\text { Frecuencia } \\
(\%)\end{array}$ \\
\hline Trimetropinsulfa & 13.6 & 9.1 & - & 22.7 \\
\hline $\begin{array}{l}\text { Ampicilina }+ \\
\text { Sulbactam }\end{array}$ & 4.5 & 9.1 & - & 13.6 \\
\hline Ampicilina & 4.5 & 4.5 & - & 9.1 \\
\hline Cefuroxima & 4.5 & 4.5 & - & 9.1 \\
\hline Eritromicina & - & - & 9.1 & 9.1 \\
\hline Ácido Nalidixico & 4.5 & - & - & 4.5 \\
\hline Norfloxacina & 4.5 & - & - & 4.5 \\
\hline $\begin{array}{l}\text { Ampicilina + } \\
\text { Acido } \\
\text { Clavulánico }\end{array}$ & - & - & 4.5 & 4.5 \\
\hline Penicilina & - & - & 4.5 & 4.5 \\
\hline Cefotaxime & - & 4.5 & - & 4.5 \\
\hline
\end{tabular}

Fuente: Archivos de la Institución de Salud. 


\section{Discusión}

En relación con los microorganismos aislados, en otra investigación similar, realizada en la ciudad de Camaguiey, Cuba (13), se encontraron los microorganismos estafilococo aureus, escherichiacoli, pseudomonas; en tanto que, en otra institución de Barranquilla, el germen más común fue el escherichiacoli, seguido de pseudomonaaeruginosa. Esto muestra una similitud entre los principales agentes causales de infección nosocomial (2). En cuanto a las diferencias encontradas en las áreas de estudio respecto a los microorganismos causantes de infecciones nosocomiales, resulta explicable que los factores que predisponen a la adquisición de una infección nosocomial son variados y van acompañados del cumplimiento de las normas de prevención de infecciones y del tipo de antibiótico utilizado.

En el seguimiento de los indicadores para el equipo de protección personal, se encontró que el tapabocas no era utilizado con la frecuencia esperada, es decir, cada vez que realizaban contacto directo con el paciente, presentándose una disconformidad a lo establecido según a literatura (14).

Se concluye que la responsabilidad estructural y formal del sistema del comité de vigilancia epidemiológica en la institución hospitalaria podría ser ejecutada de manera más eficaz, si se corrigieran factores como la poca fluidez de información actualizada de las normas, la inconstante ejecución en el seguimiento de vigilancia epidemiológica, la concientización del seguimiento de la evaluación requerida para la consecución de una posible disminución de los casos de pacientes con IN, asociada a la prevención y control de las IN, siendo este un problema generalizado entre diversas instituciones de salud (15).

Ya se ha reiterado que los microorganismos más frecuentes aislados como causa de infección nosocomial fueron escherichiacoli y pseudomonaaeuroginosa. La alta resistencia de la pseudomonaaeuroginosa puede ser explicada de manera natural y adquirida. Esto último debido a un gran número de antibióticos, como cefalosporinas de primera y segunda generación, tetraciclinas, cloranfenicol y macrólidos (16), y en concreto, por las características de su membrana celular, que tiene propiedades excepcionales de impermeabilidad. Otro factor preocupante es la capacidad de pseudomonaaeruginosa de tornarse resistente en el curso del tratamiento antibiótico. Los mismos antibióticos son capaces de inducir los mecanismos de resistencia latentes en un determinado aislamiento. Los antibióticos que se consideran con buena actividad son: las penicilinas antipseudomonas (piperacilina, ticarcilina, carbenicilina, azlocilina), asociadas a inhibidores de $\beta$-lactamasas, ceftazidima, cefepime, monobactámicos como aztreonam, carbapenémicos, quinolonas, especialmente ciprofloxacina y aminoglicósidos.

En relación al seguimiento de los indicadores, se mantuvo un alto porcentaje en el lavado de manos (entre 87.2-95.4\%); entretanto, para el equipo de protección personal y/o precaución de aislamiento, se observó un cumplimiento promedio de 45.7 a $71.6 \%$ por parte del personal de salud del hospital, siendo más alto en la UCI plena con $71.6 \%$.

Mensualmente, en los indicadores para el equipo de protección personal y/o precaución de aislamiento en las diferentes áreas, se observó el grado de cumplimiento constante de las UCIs, que estuvieron por encima de un $90 \%$. Esto puede deberse al tipo de diagnósticos de los pacientes que se encuentran en estas áreas y es acorde con políticas establecidas en otros países (17).

\section{Referencias Bibliográficas}

1. Vilar D, Mohar A, Sandoval S, De la Rosa M, Gordillo P, Volkow P. Surgicalsiteinfections at theNational Cáncer Institute in México: A casecontrol study. Am J Infect Control. 2000; 28 (1): 14-20.

2. Pérez L, Zurita I, Pérez N, Patiño N, Calvimonte O. Infecciones intrahospitalarias: agentes, manejo actual y prevención. RevCientCiencMéd. 2010; 13 (2): 90-94.

3. Sarmiento G, Rodríguez M, Barahona N, Lagares A, Cotes R, Soto J. Estudio prospectivo para describir la resistencia a antibióticos esenciales de los microorganismos aislados de pacientes hospitalizados en la Clínica de la Costa, Barranquilla, durante el periodo 2005. Revista Médica de la Costa. 2005; 1 (2): 8-41.

4. Nodarse R. Visión actualizada de las infecciones intrahospitalarias. Rev Cubana MedMilit. 2002; 31 (3): 201-208.

Cienc. innov. salud. Diciembre 2014; 2 (2):70 - 75. Universidad Simón Bolívar (Col).ISSN: 2344-8636 
5. Basulto M, Galdós M, Carr J, Díaz H. Infección nosocomial respiratoria en la Unidad de Cuidados Intensivos. Archivo Médico de Camagüey. 2009; 13 (3): 1-10.

6. Wenzel R. Preoperativeantibioticprophylaxis. N. Engl J. Med. 1992; 326 (5): 337-339.

7. Bennett J, Brachman P. Hospital Infections. Ed forth. Lippincott-Raven, 2000.

8. Navarrete S, Rangel S. Las infecciones nosocomiales y la calidad de la atención médica. Salud pública de México.1999; 41 (s1): S64-S68.

9. Morera O, González M, Guevara R, Sánchez M, Lisa O. Evaluación de los resultados de la ventilación no invasiva en una unidad emergente. AMC. 2009; 13 (4): 1-9.

10.Digiovine B, Chenowth C, Watts C, Higgins M. Theattributablemortality and costs of primary nosocomial bloodstreaminfections in theintensivecareunit. Am J. Respir. Crit. CareMed. 1999; 160 (3): 976-981.

11.Dellinger R, Levy MM, CarletJM, Bion J, Parker MM, Jaeschke R, et al. Surviving Sepsis Campaign: International guidelinesformanagement of severe sepsis and septic shock: 2008. IntensiveCareMed. 2008; 34: 17-60.

12. Almaraz S, Cabedo M, Calvelo F, Gómez C. Valoración de los inhibidores de las betalactamasas. FarmHosp 1996; 20 (4): 225-235.

13.Chediak J, Santana T, Del Águila A, Del Risco C. Caracterización de las infecciones nosocomiales en pacientes con patología oncológica. Archivo Médico de Camagüey. 2007; 11 (2): 1-7.

14.Organización Mundial de la Salud. Prevención de las infecciones nosocomiales, Guía Práctica, 2003.

15.De León R, Soto H. Infecciones Intrahospitalarias. Edición: Mc Graw Hill. México, D.C., 1996.

16.Gómez C, Leal A, Pérez $M$, Navarrete $M$. Mecanismos de resistencia en Pseudomonasaeruginosa: entendiendo a un peligroso enemigo. Rev. Fac. Med. Unal. 2005; 53 (1): 27-34.

17. Otaíza F. Políticas de control de antimicrobianos en el nivel hospitalario. Revista chilena de infectología. 2002; 19 (3): 219-221. 